\title{
RHIC Beam Energy Scan Program: Phase I and II
}

\author{
Grazyna Odyniec ${ }^{* \dagger}$ \\ Lawrence Berkeley National Laboratory, Nuclear Science Division, Berkeley, CA 94720, USA \\ E-mail: G_Odyniec@lbl.gov
}

In 2010, the Relativistic Heavy Ion Collider (RHIC) launched a multi-step experimental program to investigate the phase diagram of strongly interacting nuclear matter. The exploratory phase I of the Beam Energy Scan (BES) program was completed in 2011 with data sets ranging from 39 $\mathrm{GeV}$ down to $7.7 \mathrm{GeV}$. This, together with larger data sets at 62,130 and $200 \mathrm{GeV}$, allowed for an initial look into the uncharted territory of QCD phase diagram. This diagram is considered to be the single most important graph of our field. All measurements taken by the STAR (Solenoidal Tracker At RHIC) detector at $\sqrt{s_{N N}}$ below the RHIC injection energy are affected by large statistical errors, steeply increasing with decreasing energy. Nevertheless, they allowed for the first time a direct study of the anticipated critical point and phase transition signatures. The plans and the preparation for phase II of the BES program (in 2017-2018), with one order of magnitude larger statistics, are discussed.

8th International Workshop on Critical Point and Onset of Deconfinement,

March 11 to 15, 2013

Napa, California, USA

\footnotetext{
* Speaker.

${ }^{\dagger}$ This talk is un updated version of the presentation given at Kruger 2012 Conference in South Africa, Dec.2012.
} 


\section{Introduction}

The first decade of RHIC (Relativistic Heavy Ion Collider at Brookhaven National Laboratory, US) operation showed that nuclear matter with partonic degrees of freedom (Quark Gluon Plazma) is formed in the early stages of $\mathrm{Au}+\mathrm{Au}$ collisions at $\sqrt{s_{N N}}=200 \mathrm{GeV}$. The preliminary results revealed that this new kind of matter is very complicated and has unique and unexpected properties $[1,12,13]$. In spite of the huge recent progress in understanding the QGP phase, there are still questions to be addressed in the coming runs. In particular, the nature of the transformation from hadronic degrees of freedom to the partonic ones, and back to the state of hadron gas, is totally unknown.

So far, the theory gives very few insights into this problem. The generally accepted schematic representation of the QCD phase diagram is shown in Figure 1. Only the "edges" of this diagram are believed to be somehow understood: the lattice QCD calculations [2,3] predict a smooth crossover transition at the top RHIC energies, i.e. at critical temperature of 150-170 MeV and chemical potential $\mu_{B} \sim 0$ (top left in Fig.1), while several QCD based calculations [4, 5] show that at lower $\mathrm{T}$ and higher baryon chemical potential (right in Fig.1) a first-order phase transition may take place.

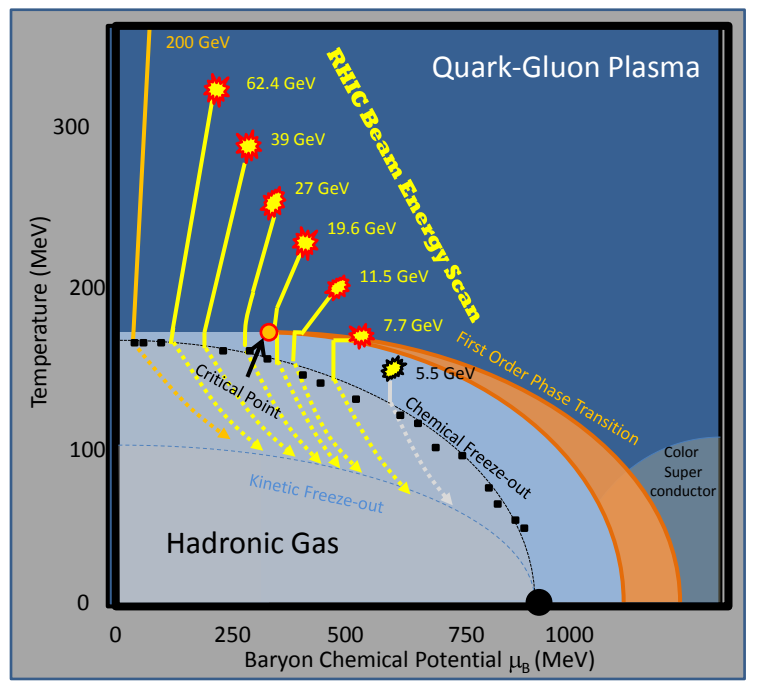

Figure 1: The picture of the QCD Phase Diagram with marked coverage of the RHIC BES program. Yellow trajectories represent schematics of the collision evolution at different energies of the BES program. The red circle symbolizes the critical point. Note, that yellow lines and the red circle are for illustrative purpose only.

The end of a first-order phase transition line marks the QCD Critical Point (CP) (the red circle in Fig.1). Although a position of the $\mathrm{CP}$ as well as a location of a first-order phase transition are not known, there is enough information coming from the lattice calculation to suggest that the most probable location of CP would be somewhere between $\mu_{B}=150$ and $500 \mathrm{MeV}$.

The $\mathrm{Au}+\mathrm{Au}$ collisions at lower, i.e. below the injection point, energies, available presently at RHIC, should be able to reach a chemical potential region of interest, and therefore allow for the exploration of the first-order phase transition, and possibly of the CP. 
In 2010 and 2011 RHIC completed phase I of the BES program with data sets at 7.7, 11.5, 19, 27 and $39 \mathrm{GeV}$. This is complemented by the data collected earlier at higher energies $(62,130$ and $200 \mathrm{GeV}$ ). Together they cover the $\mu_{B}$ interval from 20 to $450 \mathrm{MeV}$, which is believed to contain the range associated with the first order phase transition and CP.

In the following sections a short discussion of BES program specific goals, together with a few preliminary results of analyzed phase I data, will be presented. The last chapters will describe phase II, planned for 2017-2018.

\section{Beam Energy Scan Program at RHIC}

Presently the BES program is focused around three goals.

The first one, and the easiest, is to scan the phase diagram with variable collision energy (different $\mu_{B}$ and T) to find whether (and where in the $\sqrt{s_{N N}}$ ) the key QGP signatures observed at the top RHIC energy will turn off. This may suggest that system is back to the hadron gas phase. The disappearance of a single signature would not be enough to claim an onset of deconfinement, because there are other phenomena not related to deconfinement which may cause a similar effect. However, the modification or disappearance of several signatures simultaneously would definitely provide a compelling case. The particular observables identified as the essential drivers of this part of the run are: constituent quark number scaling, hadron suppression in central collisions characterized by $R_{c p}$, untriggered pair correlations in the space of pair separation in azimuth and pseudorapidity, and local parity violation in strong interactions.

The second goal is to find critical fluctuations, associated with a strong increase in the susceptibilities, which are expected in the vicinity of CP. However, because the finite size effects could wash out the critical behavior, the search for evidence of the softening of EOS as a system enters a mixed phase region implicitly associated with crossing first-order phase transition was proposed as a third goal of BES program. Essential observables in this search are: elliptic and directed flow of charged particles and of identified protons and pions, azimuthally-sensitive femtoscopy, and fluctuations indicated by large jumps in baryon, charge, and strangeness susceptibilities, as a function of system enerrgy.

Fig. 1 shows the reach of RHIC's BES program in the $\left(\mathrm{T}, \mu_{B}\right)$ plane. The yellow lines represent possible reaction trajectories at energies $\sqrt{s_{N N}}=5,7.7,11.5,19.6,27$ and $39 \mathrm{GeV}$. All energies, with the exception of $5 \mathrm{GeV}$, were run in 2010 and 2011. This choice of energies provides almost uniform coverage of the unknown $\left(\mathrm{T}, \mu_{B}\right)$ territory and may allow us to narrow down an area of interest for further study.

\section{RHIC and STAR}

RHIC has a special and unique role in the exploration of the QCD phase diagram. Even with LHC operational, RHIC remains the only facility in the world capable of providing the necessary collision energies to execute the BES program.

Out of two large experiments presently on the RHIC floor, the STAR detector with its very large and uniform acceptance in rapidity, transverse momentum and azimuth, and excellent particle 
identification capabilities due to the addition of TOF in 2010, is particularly well suited for the challenges discussed in the previous sections.

\section{Results}

In the following sections BES selected results are presented. They are grouped to represent examples of each of the three goals of the program, discussed earlier. The ones discussed here have the best resolving power given the constraints of the experiment (in phase I) and the total beam time. The details of these analyses and discussion of the other results can be found in $[6,18]$ and also in the recent Proceedings of Quark Matter Conferences [12,13], Strange Quark Matter Conference [24], and CPOD 2011 Conference [14].

\subsection{The "environment": freeze-out parameters}

First, one needs to confirm that the environment created in heavy ion collisions at BES energies is suitable for this study.

Particle multiplicities observed in heavy ion and elementary collisions have been well described by statistical-thermal models [1, 8, 18], which use experimentally measured particle ratios to extract two parameters: the temperature at the chemical freeze-out $T_{c h}$, and chemical potential $\mu_{B}$.

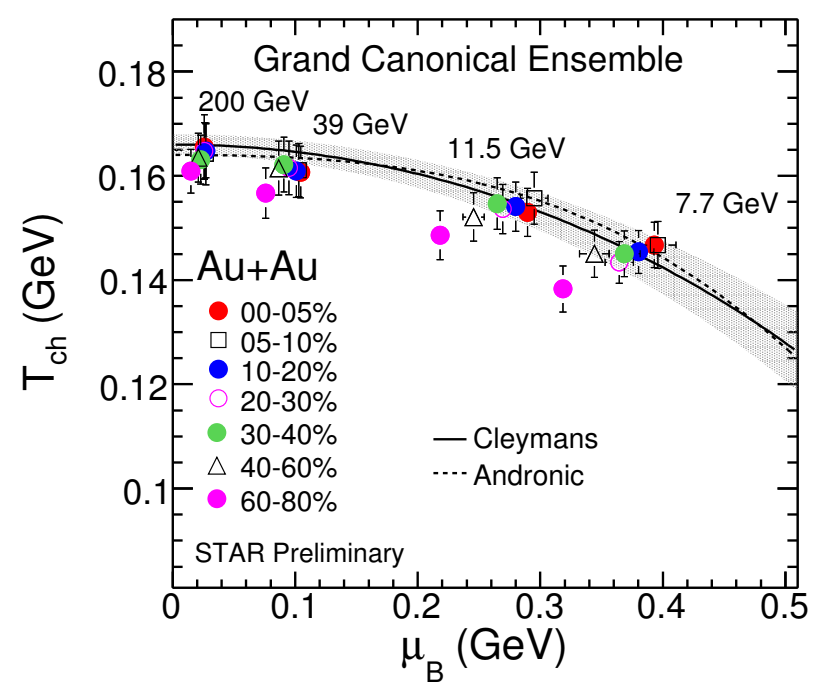

Figure 2: The variation of $T_{c h}$ with $\mu_{B}$, fitted with the Grand Canonical approach in THERMUS Model [9] using all available particle ratios, including strangeness. The lines represent systematic trends from Refs.[9, $10]$.

Fig. 2 shows the centrality dependence of the chemical freeze-out temperature as a function of chemical potential based on THERMUS model [9] with Grand Canonical ensemble for $\mathrm{Au}+\mathrm{Au}$ collisions from $200 \mathrm{GeV}$ to the lowest measured energy point at $7.7 \mathrm{GeV}$. Note, that while $T_{c h}$ decreases only slightly (from $165 \mathrm{MeV}$ at $200 \mathrm{GeV}$ to $150 \mathrm{MeV}$ at $7.7 \mathrm{GeV}$ ), there is a significant 
change in the value of $\mu_{B}$. With the lowest BES energy so far of $7.7 \mathrm{GeV}$, the RHIC $\mu_{B}$ range was extended to $400 \mathrm{GeV}$ (with about $5 \%$ error). The critical region in $\mu_{B}$ has been predicted to span on the order of $100 \mathrm{MeV}$ [11], which suggests that the overall program of BES measurements to date will offer reasonable coverage also below $\mu_{B} \sim 400 \mathrm{MeV}$ when the two recent energy points (19.6 and $27 \mathrm{GeV}$ ) are included in the analysis. The centrality dependence is still under investigation.

\subsection{Onset of the QGP}

RHIC results at top energies indicate that the passage through the phase transition to the partonic phase took place $[12,13]$. Following the evolution of the established partonic signatures with $\sqrt{s_{N N}}$ from 200 to $7.7 \mathrm{GeV}$ may bring us to the value of $\sqrt{s_{N N}}$ where these signatures change or even disappear completety. One of the key results that has been accepted as evidence of partonic degrees of freedom at RHIC has been the observation that the elliptic flow (expressed by anisotropy parameter $v_{2}$ ) scales with the number of constituent quarks in a given hadron. This indicates that the flow is established early on, where quarks are the relevant degrees of freedom (if the flow were to have been established during a hadronic phase, then the magnitude of $v_{2}$ for a given hadron would scale with its mass). Figure 3 shows the $v_{2}$ of protons and anti-protons in minimum bias collisions

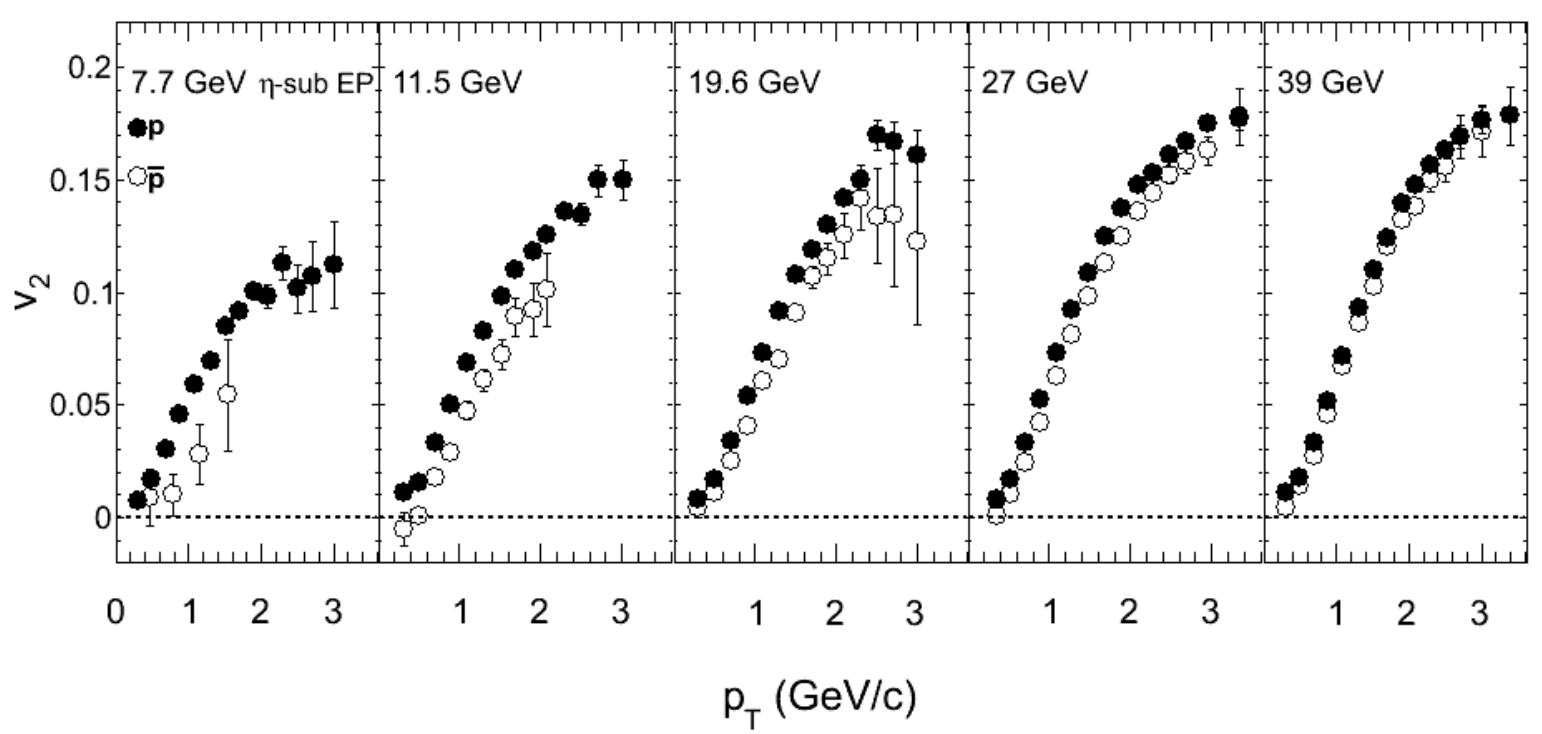

Figure 3: The $v_{2}$ of protons and anti-protons in minimum bias $\mathrm{Au}+\mathrm{Au}$ collisions as a function of $p_{t}$ for the five energies: 7.7, 11.5, 19.6, 27 and $39 \mathrm{GeV}$.

as a function of $p_{t}$ for the five energies: 7.7, 11.5, 19.6, 27 and $39 \mathrm{GeV}$. The $v_{2}$ is calculated as $\left\langle\cos 2\left(\phi-\psi_{2}\right)\right\rangle$, where $\phi$ denotes the azimuthal angle of the produced particles and $\psi_{2}$ denotes the orientation of the second-order event plane. The $v_{2}$ gradually increases with energy. At $39 \mathrm{GeV}$ the proton $v_{2}$ is only slightly higher than the anti-proton $v_{2}$, but this difference increases significantly with decreasing energy. The difference in particle and anti-particle $v_{2}$ suggests that the number 
of constituent quark (NCQ) scaling observed at $\sqrt{s_{N N}}=200 \mathrm{GeV}$ [14] may no longer be valid at lower energies. The lower the energy the stronger the violation of NCQ scaling is observed.

More insights are provided by analysis of the nuclear modification factor $\left(R_{c p}\right)$, another key signature measurement of the discovery of the QGP at RHIC. In high energy nuclear collisions the created strongly interacting medium is opaque to the fast moving partons, resulting in their measurable energy loss [15]. The suppression of high pt hadrons observed in $\mathrm{A}+\mathrm{A}$ is quantified by the variable $R_{c p}$, i.e. the properly scaled (by the number of binary collisions) ratio of the number of particles in the central $\mathrm{A}+\mathrm{A}$ collisions to the number of particles in peripheral $\mathrm{A}+\mathrm{A}$ (where a QGP is not expected to be formed). The $R_{c p}$ is equal to 1 if A-A system is a simple superposition of N-N. Any deviation from unity will imply a contribution from nuclear or medium effects. Early results on $R_{c p}$ from $200 \mathrm{GeV} \mathrm{Au+Au} \mathrm{collisions} \mathrm{at} \mathrm{RHIC} \mathrm{found} \mathrm{a} \mathrm{clear} \mathrm{suppression} \mathrm{of} \mathrm{high} p_{t}$ particles (see opened circles in Fig.4) which is attributed to the parton energy loss in the dense medium of quark gluon plasma [15]. The disappearance of this suppression would imply the absence of the partonic energy loss caused by the presence of quark gluon plasma, and would suggest that the hadronic interactions become dominant (replacing partonic ones). Thus, the study of the $R_{c p}$ evolution with energy becomes very important. The results on $R_{c p}$ of charged particles from $\mathrm{Au}+\mathrm{Au}$ collisions at $\sqrt{s_{N N}}=7.7,11.5,19.6,27$ and $39 \mathrm{GeV}$, together with the points for 62.4 and $200 \mathrm{GeV}$, are presented in Fig.4. The $p_{t}$ reach of the charged hadron spectra extends beyond $3 \mathrm{GeV} / \mathrm{c}$ for all energies. The figure shows dramatic change in the suppression pattern. There is clear evidence of suppression at $\sqrt{s_{N N}}=39,62.4$ and $200 \mathrm{GeV}$, which then appears to turn off between 19.6 and 39 $\mathrm{GeV}$, becoming an enhancement at 7.7 and $11.5 \mathrm{GeV}$.

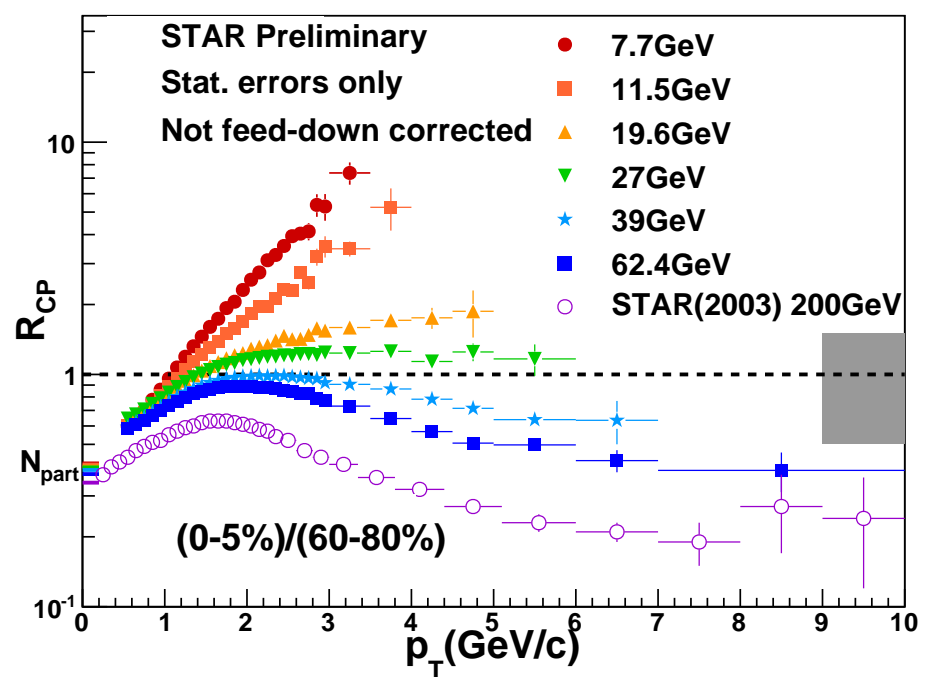

Figure 4: The STAR preliminary results of the energy dependence of charged particles $R_{c p}$ [16]. The error bars on points reflect statistical errors. The systematic error (gray box) is independent of energy and $p_{t}$, and combines error on $N_{\text {coll }}$ scaling and on the yields due to the background.

Those observations together with other ones [12,13], suggest clearly that the change of dominant degrees of freedom, from partonic to hadronic, takes place somewhere in the energy range between 11.5 and $39 \mathrm{GeV}$. This interval of $\sqrt{s_{N N}}$ becomes the main focus phase II of the BES program, which is scheduled to start in 2017. 


\subsection{Critical Point (CP)}

The location of the critical point is the most anticipated discovery of the BES program. Lattice QCD [17] shows an increase of susceptibilities of conserved quantities such as baryon number $(B)$, charge $(Q)$, and strangeness $(S)$ at the critical point (a similar critical behavior is known from classic thermodynamics), which translate into critical fluctuations in the multiplicity distributions that can be studied experimentally. The key observation is a change of the observable as a function of chemical potential $\mu_{B}$. Therefore the experimental strategy amounts to small changes in energy with careful measurement of all aspects of the fluctuations. The STAR experiment studies of eventby-event fluctuation of proton, pion and kaon multiplicity ratios $(K / \pi, p / \pi$ and $K / p)$ in $\mathrm{Au}+\mathrm{Au}$ collisions at $\sqrt{s_{N N}}=7.7,11.5,19.6,39$ and $200 \mathrm{GeV}$ do not show anomalous behavior. All measured ratios change smoothly with energy or there is no observed change at all.

It has been noted that the multiplicity distribution moment analysis would be more suitable to these studies. Typically, experimental investigations are limited to the second moments, which are proportional to the square of the correlation length. Those, however, are estimated to be quite small in the vicinity of the critical point $(\sim 2-3 \mathrm{fm})$ [18]. Therefore, the higher moments of eventby-event multiplicity distribution are used; the higher the order of the moment the more sensitive it is to the correlation length ( $\xi$ ) of the system, e.g. the third moment (skewness) $s \sim \xi^{4.5}$ and the fourth moment (kurtosis) $\kappa^{2} \sim \xi^{7}[18]$.

The measurements of higher moments of event-by-event identified particle multiplicity distributions and their variation with centrality and beam energy provide the very first direct link between experimental observables and Lattice QCD calculations. The measurements of higher moments of the net-proton distribution (proxy for net-baryon) carried out by the STAR collaboration are presented in Fig.5, with the $S \sigma$ and $\kappa \sigma^{2}$ of net-proton distributions in four centrality bins: 0-5\%, $5-10 \%, 30-40 \%$ and $70-80 \%$ in $\mathrm{Au}+\mathrm{Au}$ collisions [19, 20, 25]. In the bottom panel, the ratios of $S \sigma$ over the corresponding Poisson expectations are shown. As one can see in the middle and bottom panel, the $\kappa \sigma^{2}$ and normalized $S \sigma$ values are close to the Poisson expectation for $\mathrm{Au}+\mathrm{Au}$ collisions above $\sqrt{s_{N N}}=39 \mathrm{GeV}$. At lower energies the results from most central collisions, $0-5 \%$, seem to deviate from Poisson, especially at $\sqrt{s_{N N}}=19.6 \mathrm{GeV}$ central collisions (middle and bottom panel of Fig.5). At lower beam energy, large statistical errors preclude any decisive conclusions at the present time.

The energy dependent results from transport model UrQMD [21], the dot-dashed line in Fig.5, which represent "non-CP scenario" (including baryon number conservation and hardon scattering etc.) do not reproduce data. They show a smooth decreasing trend from high to low energy.

\subsection{First-order phase transition}

Direct flow $\left(v_{1}\right)$ probes the early stage of the collisions and is sensitive to the compression. Therefore, it is expected to be also sensitive to the first-order phase transition. The change of sign in the slope of $d v_{1} / d y$ for protons has been proposed, based on hydrodynamic model calculations [22], to be a probe to the softening of the equation of state (EOS) and/or the first order phase transition. According to these calculations a first-order phase transition leads to a softening of the EOS, and this in turn leads to a local minimum in directed flow slope as a function of energy. This 


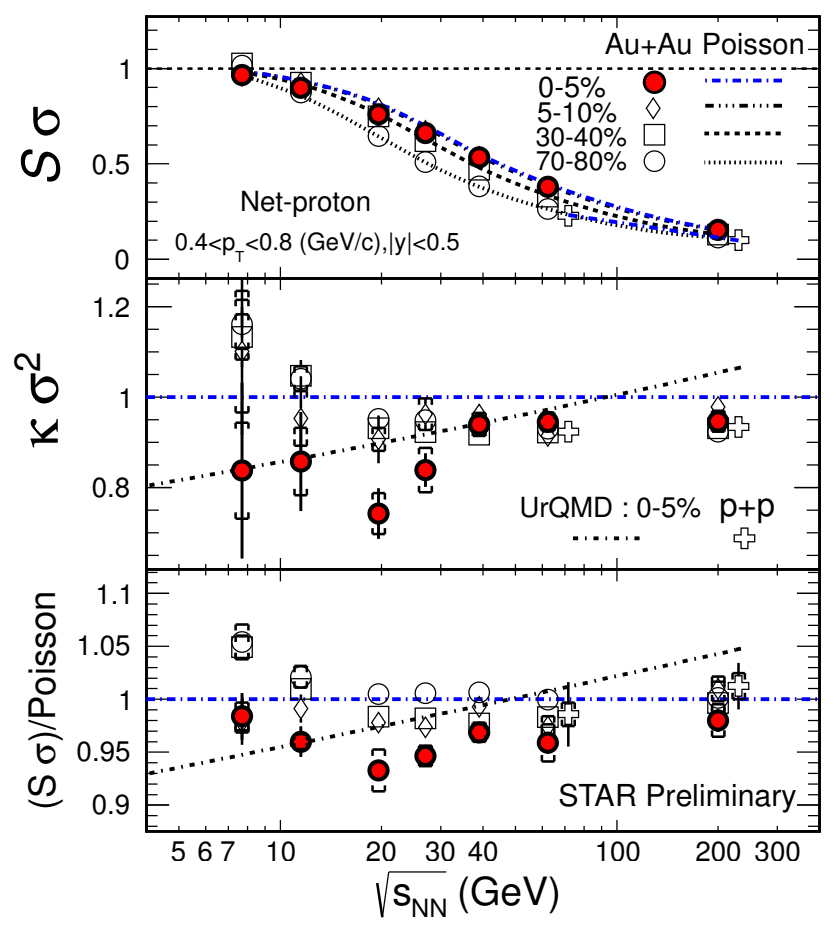

Figure 5: STAR preliminary results of the energy and centrality dependence of $S \sigma$ (top panel), $\kappa \sigma^{2}$ (middle panel) and $S \sigma /$ Poisson (bottom panel) of net-proton distributions from Au+Au collisions at RHIC [19]. Results from $\mathrm{p}+\mathrm{p}$ collisions at $\sqrt{s_{N N}}=62.4 \mathrm{GeV}$ and $200 \mathrm{GeV}$ are shown as crosses. The results are compared to UrQMD model calculations for the top 5\% central collisions shown as the dotted line. Dotdashed lines are expectations from Poisson distributions.

phenomenon is referred in the literature as a "collapse of proton flow" and the minimum is expected to correspond to the "softest point" in the EOS, where a relatively long-lived mixed phase occurs.

The direct flow excitation function measured by STAR for protons, antiprotons and pions near mid-rapidity is presented in Fig.6. The plotted quantity is $d v_{1} / d y^{\prime}$, where the primed quantity $y^{\prime}$ refers to normalized rapidity $y / y_{\text {beam }}$. The lower panel in Fig. 6 reveals that the inferred $v_{1}$ slope for net protons (the net protons signal is obtained by subtracting properly scaled antiprotons from protons) becomes negative with good statistical significance at 11.5 and $19.6 \mathrm{GeV}$, while it is consistent with zero at $27 \mathrm{GeV}$ and positive at $7.7 \mathrm{GeV}$ and above $27 \mathrm{GeV}$, including at $200 \mathrm{GeV}$. Indeed, the prominent dip and its associated double sign change bears a striking resemblance to the predicted signatures of a softening of the EOS associated with the first order phase transition [22]. In contrast, there is no hint of this remarkable behavior in the UrQMD transport model (purple line in Fig.6) which shows a positive slope at all energies for this observable. To better understand this observation and the possible role and relevance of stopping, one needs to make measurements of net proton $v_{1}$ as a function of centrality [23]. This will be possible in BES phase II part of the program which will deliver an order of magnitude more statistics. 


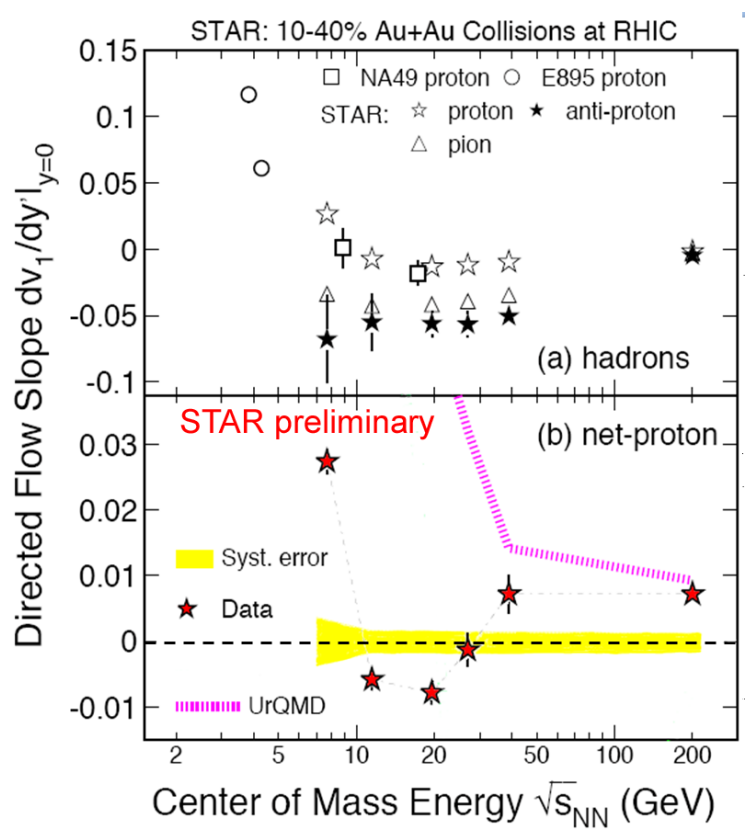

Figure 6: (a) - slope of directed flow vs rapidity for protons, antiprotons, and pions as a function of beam energy. (b) - proton signal with antiproton signal subtracted after appropriate scaling. The prediction of the UrQMd transport model is also plotted.

\section{Summary}

Phase I of the BES program allowed to extend the $\mu_{B}$ reach of RHIC facility to values of approximately $400 \mathrm{MeV}$. Both the collider and experiment performances were excellent. As expected, a number of very interesting results was reported. It was observed that hadronic interactions in the produced nuclear matter become dominant over partonic interactions, when the collision energy decreases, especially below $\sqrt{s_{N N}}=11.5 \mathrm{GeV}$. In the most central Au+Au collisions, a deviation of the products of moments of net-protons distribution from the Poissonian expectations around $\mu_{B}$, corresponding to beam energies of $19.6-27 \mathrm{GeV}$ was found. The first hints of the softening of the EOS were noticed: the behavior of the directed flow, $v_{1}$, of charged hadrons at $\sqrt{s_{N N}}=7.7$ - $200 \mathrm{GeV}$ resembles striking similarity to the hydro-model predictions with the first-order phase transition built in. The implications of these measurements to understand the QCD phase structure are not yet resolved. The statistics collected during phase I are not sufficient to complete those studies. Therefore, STAR proposes the BES phase II program of precision measurements to map out a QCD phase diagram with an order of magnitude increase in data samples. BES phase II is planed for 2017.

\subsection{BES phase II}

The installation of electron cooling in RHIC will provide a significant improvement in luminosity for collisions below $\sqrt{s_{N N}}$ of $20 \mathrm{GeV}$. Simulation results indicate that at 7 and $20 \mathrm{GeV}$, the expected increasing factors are about 3-5 and 10, respectively [26]. Recently [27], it was shown that an additional improvement in luminosity may be possible by operating with longer bunches at 
the space-charge limit in a collider, which further extends benefits from cooling at low energies. Overall improvement from cooling could be about a 10-fold gain in total luminosity. A major additional improvement in BES phase II analysis will be provided by the instrumentation of the inner sectors of the STAR TPC (so called "iTPC upgrade"), which will result in significant extention of $y$ and $p_{t}$ range of experiment. The search for both the critical point and the onset of deconfinement will benefit substantially from the larger rapidity acceptance and lower $p_{t}$ theshold.

\subsection{Fixed target mode}

While the electron cooling in RHIC and iTPC upgrade are planned only for 2017, the extension of the BES program to the lower energies via the fixed target mode will be tested already in 2014.

Running the STAR in fixed target mode would allow for extension of the range of accessible baryon chemical potential from the current maximum of about $\mu_{B} \sim 400 \mathrm{MeV}$ up to $\sim 800 \mathrm{MeV}$ (corresponding to the collision energy $\sqrt{s_{N N}} \sim 2.5 \mathrm{GeV}$ ), in the QCD phase diagram. This will allow for study of the QCD phase diagram in the region of compressed baryonic matter and to investigate regions bellow the suggested energy of the onset of deconfinement. The fixed target mode will provide an extension of the BES II program down to beam energies significantly below the lower limit of RHIC operations in the collider mode.

The fixed target will be mounted inside RHIC beam pipe. The fixed target data-taking can proceed with minimal or no impact on RHIC operations in collider mode. Collisions between injection-energy $(9.8 \mathrm{GeV})$ gold ions and a fixed gold target will allow the study of $\sqrt{s_{N N}}=4.5$ $\mathrm{GeV}$. The already developed sub-injection energy beams at RHIC, 11.5 and $7.7 \mathrm{GeV}$, will provide fixed target collisions at energies of $\sqrt{s_{N N}}=3.5$ and $3.0 \mathrm{GeV}$, respectively. And the proposed for 2014 run at $14.6 \mathrm{GeV}$ will provide $\sqrt{s_{N N}}=4.0 \mathrm{GeV}$ fixed target collisions. The installation of $1 \%$ Au target in the STAR experiment is planned for the summer 2013 shutdown. It will be located about 2 meters from the center of the detector. During gold running, fixed-target events will be taken at injection energy at the beginning of each fill. This will happen concurrently with $\mathrm{Au}+\mathrm{Au}$ data-taking at $200 \mathrm{GeV}$ (i.e. during every store prior to accelerating Au ions to $200 \mathrm{GeV}$ ). The BES phase II program, which is discussed above, requests $\mathrm{Au}+\mathrm{Au}$ collisions at 19.6, 14.6, 11.5 and 7.7 $\mathrm{GeV}$ after electron cooling upgrade. The gold target will be in place for these runs, allowing fixed target collisions at 4.5, 4.0,3.5, and $3.0 \mathrm{GeV}$, respectively. These events can be triggered upon and recorded without reducing the number of collider events recorded, as the expected luminosities will not fill the DAQ bandwidth.

\section{Concluding remarks}

RHIC sits at a "sweet spot" in energy, in which rapid changes occur in a number of signatures for energies up to approximately $30 \mathrm{GeV}$, while remaining surprisingly stable beyond that over the two orders of magnitude to the LHC.

With BES phase II the most exciting physics is still to come.

\section{References}

[1] J.Adams et al., (STAR Coll.), Nucl. Phys. A757, 102 (2005) 
[2] F.Karsch et al., Nucl. Phys. Proc. Suppl. 129, 614 (2004)

[3] M.Cheng et al., Phys. Rev.D 79, 074505 (2009) and references therein

[4] Y.Aoki et al. Nature 443, 675 (2006)

[5] S.Ejiri, Phys. Rev. D78, 074507 (2008), E.S.Bowwman and J.I.Kapusta, Phys. Rev. C79, 015202 (2009)

[6] http://drupal.star.bnl.gov/STAR/starnotes/public/sn0494

[7] B.I.Abelev et al., (STAR Coll.), Phys. Rev. C79, 034909 (2009); Phys. Rev. C81, 024911 (2010)

[8] P.Braun-Munzinger et al., Phys. Lett. B344, 43 (1995)

[9] S.Wheaton, J.Cleymans, and M.Hauer, Comp. Phys. Communications 180, 84 (2009)

[10] A.Andronic et al., Nucl. Phys. A 834, 237 (2010)

[11] R.V.Gavai and S.Gupta, Phys. Rev. D78, 114503 (2008); Phys. Rev. D71, 114014 (2005); arXiv:1001.3796; S.Gupta, arXiv:0909.4630; M.Asakawa et al., Phys. Rev. Lett. 101, 122302 (2008); P.Costa et al., Europhys. Lett. 86, 31001 (2009); Phys. Lett. B647, 431 (2007)

[12] Proceedings of Quark Matter 2011 Conference, Annecy, France, May 2011, J.Phys.G: Nucl.Part.Phys. 38 (2011)

[13] Proceedings of Quark Matter 2012 Conference, August 2012, Washington DC, US.

[14] L.Adamczyk et al., (STAR Coll.), arXiv: 1301.2348, submitted to PRC (in press), arXiv:1301.2347, submitted to PRL (in press); A.Schmah et al., (STAR Coll.), J.Phys.G: Nucl. Part. Phys. 38, 124045 (2011), Central Eur.J.Phys. 10, 1238 (2012), arXiv:1202.2389; S.Shi et al., (STAR Coll.),Central Eur. J. Phys. 10,(2012)

[15] X.N.Wang and M.Gyulassy, Phys. Rev. D44, 3501 (1991)

[16] E.Sangaline et al., (STAR Coll.), Quark Matter 2012 Conference Proceedings, Washington DC, August, 2012.

[17] M.A.Stephanov, Phys. Rev. Lett. 102, 032301 (2009); Phys. Rev. Lett. 107, 052301 (2001); M.Cheng et al., Phys. Rev. D79, 074505 (2009); R.V.Gavai and S.Gupta, Phys.Lett. B696, 459 (2011).

[18] M.A. Stephanov, arXiv:0809.3450.

[19] X.F.Luo et al., (STAR Coll.) Quark Matter 2012 Conference Proceedings, Washington DC, August, 2012.

[20] X.Dong et al.,(STAR Coll.) Quark Matter 2012 Conference Proceedings, Washington DC, August, 2012; D.Cebra at al.,(STAR Coll.) Quark Matter 2012 Conference Proceedings, Washington DC, August, 2012.

[21] UrQMD: http://urqmd.org/

[22] H.Stocker, Nucl. Phys. A750, 121 (2005); L.P.Csernai and D.Rohrich, Phys. Lett. B 458, 454 (1999); J.Brachmann et al., Phys. Rev. C61, 24909 (2000); D.R.Rischke et al., Heavy Ion Phys. I, 309 (1995)

[23] D. Keane et al., (STAR Coll.) Proc.Xth Quark Confinement and the Hadron Spectrum, Munich, October 2012, PoS (in press).

[24] S.Kabana et al., (STAR Coll.), Acta Phys. Pol. 2011, arXiv:1203.1814.

[25] M.M.Aggarwal et al., (STAR Coll.), Phys. Rev. Lett. 105 (2010) 022302.

[26] A.Fedotov and W.Fischer, RHIC CA-D, private communications, 2012.

[27] A.Fedotov and /m.Blaskiewicz, BNL C-AD Tech Note: C-A/AP/449 (February, 2012) 